\title{
Établir les priorités de la vaccination contre la COVID-19 en fonction de la prévalence des facteurs de risque chez les adultes au Canada
}

\author{
Finlay A. McAlister MD MSc, Tracey Bushnik MBA, Alexander A. Leung MD MSP, Lynora Saxinger MD
}

Citation : CMAJ 2021 April 26;193:E617-21. doi : 10.1503/cmaj.210529-f; diffusion hâtive le 9 avril 2021

Voir la version anglaise de l'article ici : www.cmaj.ca/lookup/doi/10.1503/cmaj.210529

C ompte tenu de la demande mondiale, de la fabrication centralisée et des limites de l'infrastructure de livraison et d'administration des vaccins au Canada, plusieurs s'inquiètent d'un possible déséquilibre entre l'offre et la demande de vaccins contre le coronavirus du syndrome respiratoire aigu sévère 2 (SRAS-CoV-2). Or, l'établissement d'une stratégie optimale d'administration des vaccins afin de maximiser les avantages pour la santé publique au Canada n'est pas un processus simple. La première phase du déploiement du vaccin au Canada était axée sur les personnes les plus susceptibles d'être infectées par le SRAS-CoV-2 ou de le transmettre, soit les résidents et le personnel des établissements de soins de longue durée, les personnes âgées, les adultes des communautés autochtones et les travailleurs de la santé de première ligne $^{1}$. Cependant, le débat se poursuit pour ce qui est de déterminer s'il faut définir les prochaines étapes de la vaccination (étapes 2 et 3 du schéma du Comité consultatif national de l'immunisation $[\mathrm{CCNI}])^{2}$ uniquement en fonction de l'âge (l'âge étant le plus important facteur de risque associé à un mauvais pronostic de la maladie à coronavirus 2019 [COVID-19]), ou s'il est préférable de procéder à un déploiement plus complexe ciblant les personnes présentant d'autres facteurs de risque importants (p. ex., des facteurs de risque médicaux ou liés à l'exposition) $)^{3}$. S'ajoute au débat sur la vaccination prioritaire la question du report de l'administration des deuxièmes doses pour permettre au plus grand nombre possible de personnes de recevoir au moins une dose $\mathrm{e}^{3,4}$. Les approches actuelles en matière de vaccination varient considérablement d'une province et d'un territoire à l'autre, tout comme les définitions des personnes considérées "à haut risque » sur le plan médical, géographique ou professionnel ${ }^{5}$.

Afin d'optimiser les stratégies de vaccination, nous devons d'abord déterminer la proportion d'adultes canadiens qui présentent un risque accru de complications graves en cas d'infection par le SRAS-CoV-2. Une évaluation récente des données de la National Health and Nutrition Examination Survey

\section{POINTS CLÉS}

- Le débat se poursuit sur la meilleure façon d'établir les priorités de la vaccination contre le SRAS-CoV-2 une fois que les adultes âgés les plus vulnérables auront été vaccinés.

- Bien que l'âge soit le facteur de risque le plus important associé à une forme grave de la COVID-19, les trois quarts des adultes au Canada présentent au moins un autre facteur de risque de maladie grave, et un tiers en présentent 2 ou plus, selon l'analyse des données d'une enquête nationale.

- Il est nécessaire d'adopter une approche plus nuancée dans l'établissement des priorités en matière de vaccination, qui tient compte de la prévalence des facteurs de risque et de la pondération à accorder à chacun, ainsi que de l'exposition aux risques géographiques et professionnels.

- Il faudra continuer de respecter les recommandations de la santé publique concernant le port du masque universel, la distanciation physique et le lavage fréquent des mains durant le déploiement des programmes de vaccination, étant donné la forte prévalence des facteurs de risque dans la population canadienne.

- Les politiques qui autorisent l'allongement de l'intervalle entre la première et la deuxième dose de vaccin dans le but de favoriser l'administration rapide d'une première dose au plus grand nombre de personnes possible sont également judicieuses, compte tenu du lourd fardeau que représentent les facteurs de risque, dans la mesure où les données sur la protection conférée par la première dose demeurent favorables.

(NHANES) a révélé que les trois quarts des adultes aux ÉtatsUnis présentaient au moins un facteur de risque d'une forme grave de la COVID-19, selon la définition des Centers for Disease Control and Prevention (CDC) des États-Unis ${ }^{6}$. La présente analyse examine le fardeau des facteurs de risque dans la population canadienne; elle se veut un complément aux analyses des ensembles de données administratives provinciales et vise à mieux éclairer l'établissement des priorités de la vaccination contre le SRAS-CoV-2 au Canada. 
Quelle est la fréquence des facteurs de risque d'une forme grave de la COVID-19 chez les adultes vivant dans la collectivité au Canada?

Bien que les provinces et territoires du Canada soient en mesure d'évaluer leurs données administratives sur la santé afin de déterminer la proportion de leur population courant un risque de développer une forme grave de la COVID-19, leur capacité à estimer la fréquence des maladies est limitée : en effet, l'utilisation de ces données entraine une sous-estimation du fardeau des maladies chroniques qui sont principalement prises en charge en consultation externe ${ }^{7,8}$. De plus, certains facteurs de risque associés aux formes graves de la COVID-19 (p. ex., le tabagisme, l'obésité, l'origine ethnique) ${ }^{9-14}$ ne sont pas pris en compte. Par conséquent, nous avons estimé la prévalence des facteurs de risque associés à une forme grave de la COVID-19 chez les adultes vivant dans la collectivité au Canada à partir de 2 enquêtes basées sur la population : l'Enquête sur la santé dans les collectivités canadiennes $(E S C C)^{15}$ de 2019 et l'Enquête canadienne sur les mesures de la santé (ECMS) ${ }^{16}$ de 2016 à 2019. Nous avons défini les facteurs de risque associés à une forme grave de la COVID-19 d'après l'algorithme de prédiction du risque évolutif QCOVID, basé sur les données de 8,2 millions d'adultes en Angleterre ${ }^{10}$. L'algorithme QCOVID inclut tous les facteurs de risque de formes plus graves de la COVID-19 qui ont été définis par les $\mathrm{CDC}^{6}$ et dans une revue rapide ${ }^{11}$ menée pour le CCNI. Bien que le calculateur QCOVID fournisse des estimations du risque

Tableau 1 (partie 1 de 2) : Prévalence pondérée des facteurs de risque associés à une forme grave de la CoviD-19 chez les répondants adultes à l'Enquête sur la santé dans les collectivités canadiennes de 2019 dans les provinces du Canada, par sexe et par groupe d'âge

\begin{tabular}{|c|c|c|c|c|c|c|c|c|c|c|c|c|}
\hline \multirow[b]{2}{*}{$\begin{array}{l}\text { Facteur de } \\
\text { risque }\end{array}$} & \multicolumn{2}{|c|}{$18-49$ ans } & \multicolumn{2}{|c|}{ 50-59 ans } & \multicolumn{2}{|c|}{$60-69$ ans } & \multicolumn{2}{|c|}{70 ans et plus } & \multicolumn{2}{|c|}{ Population totale } & \multicolumn{2}{|c|}{$\begin{array}{l}\text { HR ajusté (IC à } 95 \% \text { ) } \\
\text { pour les formes graves } \\
\text { de la CovID-19* }\end{array}$} \\
\hline & $\begin{array}{l}\text { Hommes } \\
\text { (IC à } 95 \%) \\
\text { (\%) }\end{array}$ & $\begin{array}{l}\text { Femmes } \\
\text { (IC à } 95 \%) \\
\text { (\%) }\end{array}$ & $\begin{array}{l}\text { Hommes } \\
\text { (IC à } 95 \%) \\
\text { (\%) }\end{array}$ & $\begin{array}{l}\text { Femmes } \\
\text { (IC à } 95 \%) \\
(\%)\end{array}$ & $\begin{array}{l}\text { Hommes } \\
\text { (IC à } 95 \%) \\
\text { (\%) }\end{array}$ & $\begin{array}{l}\text { Femmes } \\
\text { (IC à } 95 \%) \\
\text { (\%) }\end{array}$ & $\begin{array}{l}\text { Hommes } \\
\text { (IC à } 95 \%) \\
\text { (\%) }\end{array}$ & $\begin{array}{l}\text { Femmes } \\
\text { (IC à } 95 \%) \\
\text { (\%) }\end{array}$ & $\begin{array}{l}\text { Hommes } \\
\text { (IC à } 95 \%) \\
\text { (\%) }\end{array}$ & $\begin{array}{l}\text { Femmes } \\
\text { (IC à } 95 \%) \\
(\%)\end{array}$ & Hommes & Femmes \\
\hline $\begin{array}{l}\text { Revenu du } \\
\text { ménage } \\
\text { (quintile } \\
\text { inférieur)† }\end{array}$ & $\begin{array}{c}19,4 \\
(18,1-20,7)\end{array}$ & $\begin{array}{c}23,1 \\
(21,8-24,4)\end{array}$ & $\begin{array}{c}15,1 \\
(13,2-17,3)\end{array}$ & $\begin{array}{c}14,9 \\
(13,2-16,8)\end{array}$ & $\begin{array}{c}15,6 \\
(14,1-17,2)\end{array}$ & $\begin{array}{c}20,2 \\
(18,4-22,1)\end{array}$ & $\begin{array}{c}20,1 \\
(18,6-21,8)\end{array}$ & $\begin{array}{c}29,4 \\
(27,7-31,1)\end{array}$ & $\begin{array}{c}18,2 \\
(17,3-19)\end{array}$ & $\begin{array}{c}22,2 \\
(21,4-23,1)\end{array}$ & $\begin{array}{c}1,5 \\
(1,4-1,6) \text { ฯ }\end{array}$ & $\begin{array}{c}1,5 \\
(1,4-1,6)\end{array}$ \\
\hline $\begin{array}{l}\text { Tabagisme } \\
\text { (quotidien ou } \\
\text { occasionnel) }\end{array}$ & $\begin{array}{c}20,7 \\
(19,6-22,0)\end{array}$ & $\begin{array}{c}13,7 \\
(12,8-14,6)\end{array}$ & $\begin{array}{c}21,0 \\
(19,1-23,2)\end{array}$ & $\begin{array}{c}17,0 \\
(15,4-18,7)\end{array}$ & $\begin{array}{c}17,1 \\
(15,6-18,8)\end{array}$ & $\begin{array}{c}14,4 \\
(13,0-15,9)\end{array}$ & $\begin{array}{c}7,6 \\
(6,7-8,6)\end{array}$ & $\begin{array}{c}5,9 \\
(5,3-6,7)\end{array}$ & $\begin{array}{c}18,5 \\
(17,7-19,3)\end{array}$ & $\begin{array}{c}13,2 \\
(12,5-13,8)\end{array}$ & $\begin{array}{r}2, \\
(1,5-3, \\
30 \text { paquets } \\
\text { plus (pas de } \\
\text { par } 5\end{array}$ & $\begin{array}{l}2 \\
\text { L) pour } \\
\text {-années ou } \\
\text { répartition } \\
\text { exe) }\end{array}$ \\
\hline Diabète & $\begin{array}{c}2,9 \\
(2,4-3,5)\end{array}$ & $\begin{array}{c}1,7 \\
(1,3-2,1)\end{array}$ & $\begin{array}{c}11,7 \\
(10,0-13,7)\end{array}$ & $\begin{array}{c}6,4 \\
(5,3-7,7)\end{array}$ & $\begin{array}{c}18,1 \\
(16,4-19,9)\end{array}$ & $\begin{array}{c}12,0 \\
(10,7-13,5)\end{array}$ & $\begin{array}{c}24,8 \\
(23,1-26,5)\end{array}$ & $\begin{array}{c}16,5 \\
(15,1-18,0)\end{array}$ & $\begin{array}{c}9,7 \\
(9,2-10,3)\end{array}$ & $\begin{array}{c}6,5 \\
(6,0-6,9)\end{array}$ & $\begin{array}{c}2,7 \\
(2,4-3,0)\end{array}$ & $\begin{array}{c}2,7 \\
(2,4-3,2)\end{array}$ \\
\hline \multicolumn{13}{|l|}{ Obésité } \\
\hline$I M C \geq 30$ & $\begin{array}{c}19,4 \\
(18,3-20,5)\end{array}$ & $\begin{array}{c}18,6 \\
(17,5-19,8)\end{array}$ & $\begin{array}{c}27,8 \\
(25,5-30,1)\end{array}$ & $\begin{array}{c}22,9 \\
(20,8-25,2)\end{array}$ & $\begin{array}{c}25,5 \\
(23,7-27,5)\end{array}$ & $\begin{array}{c}24,2 \\
(22,3-26,1)\end{array}$ & $\begin{array}{c}18,7 \\
(17,3-20,3)\end{array}$ & $\begin{array}{c}18,1 \\
(16,8-19,5)\end{array}$ & $\begin{array}{c}21,7 \\
(20,9-22,6)\end{array}$ & $\begin{array}{c}20,2 \\
(19,4-21,0)\end{array}$ & \multicolumn{2}{|c|}{$\begin{array}{c}1,8 \\
(1,4-2,4)^{\star \star} \text { (pas de } \\
\text { répartition par sexe) }\end{array}$} \\
\hline $\begin{array}{l}\text { IMC de } 30 \text { à } \\
39,9\end{array}$ & $\begin{array}{c}17,3 \\
(16,2-18,4)\end{array}$ & $\begin{array}{c}15,6 \\
(14,5-16,6)\end{array}$ & $\begin{array}{c}25,9 \\
(23,7-28,1)\end{array}$ & $\begin{array}{c}20,1 \\
(18,1-22,3)\end{array}$ & $\begin{array}{c}24,1 \\
(22,3-26,1)\end{array}$ & $\begin{array}{c}21,5 \\
(19,7-23,4)\end{array}$ & $\begin{array}{c}17,9 \\
(16,5-19,4)\end{array}$ & $\begin{array}{c}15,9 \\
(14,7-17,3)\end{array}$ & $\begin{array}{c}19,9 \\
(19,2-20,7)\end{array}$ & $\begin{array}{c}17,4 \\
(16,6-18,1)\end{array}$ & \multicolumn{2}{|c|}{$\begin{array}{c}1,8 \\
(1,5-2,2) \dagger \dagger \text { (pas de } \\
\text { répartition par sexe) }\end{array}$} \\
\hline$I M C \geq 40$ & $\begin{array}{c}2,1 \\
(1,7-2,6)\end{array}$ & $\begin{array}{c}3,1 \\
(2,6-3,7)\end{array}$ & $\begin{array}{c}1,9 \\
(1,3-2,7)\end{array}$ & $\begin{array}{c}2,8 \\
(2,2-3,6)\end{array}$ & $\begin{array}{c}1,4 \\
(1,0-1,9)\end{array}$ & $\begin{array}{c}2,7 \\
(2,0-3,5)\end{array}$ & $\begin{array}{c}0,8 \\
(0,6-1,2)\end{array}$ & $\begin{array}{c}2,1 \\
(1,7-2,7)\end{array}$ & $\begin{array}{c}1,8 \\
(1,5-2,1)\end{array}$ & $\begin{array}{c}2,8 \\
(2,5-3,2)\end{array}$ & \multicolumn{2}{|c|}{$\begin{array}{c}2,5 \\
(1,8-3,4) \dagger \dagger \text { (pas de } \\
\text { répartition par sexe) }\end{array}$} \\
\hline Cardiopathies & $\begin{array}{c}0,8 \\
(0,6-1,1)\end{array}$ & $\begin{array}{c}0,7 \\
(0,5-1,0)\end{array}$ & $\begin{array}{c}5,6 \\
(4,5-6,9)\end{array}$ & $\begin{array}{c}2,6 \\
(2,0-3,3)\end{array}$ & $\begin{array}{c}11,7 \\
(10,3-13,3)\end{array}$ & $\begin{array}{c}5,3 \\
(4,5-6,3)\end{array}$ & $\begin{array}{c}20,6 \\
(19,1-22,2)\end{array}$ & $\begin{array}{c}13,6 \\
(12,5-14,7)\end{array}$ & $\begin{array}{c}6,0 \\
(5,6-6,4)\end{array}$ & $\begin{array}{c}3,8 \\
(3,5-4,1)\end{array}$ & $\begin{array}{c}1,2 \\
(1,1-1,3)\end{array}$ & $\begin{array}{c}1,3 \\
(1,2-1,4)\end{array}$ \\
\hline MPOC $\ddagger$ & $\begin{array}{c}1,5 \\
(1,0-2,3)\end{array}$ & $\begin{array}{c}1,0 \\
(0,7-1,4)\end{array}$ & $\begin{array}{c}2,8 \\
(2,2-3,6)\end{array}$ & $\begin{array}{c}3,1 \\
(2,5-3,8)\end{array}$ & $\begin{array}{c}5,8 \\
(4,7-7,1)\end{array}$ & $\begin{array}{c}5,9 \\
(5,1-6,9)\end{array}$ & $\begin{array}{c}7,8 \\
(6,9-8,7)\end{array}$ & $\begin{array}{c}8,0 \\
(7,2-8,8)\end{array}$ & $\begin{array}{c}3,9 \\
(3,5-4,4)\end{array}$ & $\begin{array}{c}4,1 \\
(3,8-4,4)\end{array}$ & $\begin{array}{c}1,3 \\
(1,2-1,4)\end{array}$ & $\begin{array}{c}1,3 \\
(1,2-1,4)\end{array}$ \\
\hline Asthme & $\begin{array}{c}6,6 \\
(5,9-7,4)\end{array}$ & $\begin{array}{c}9,4 \\
(8,6-10,2)\end{array}$ & $\begin{array}{c}6,2 \\
(5,2-7,5)\end{array}$ & $\begin{array}{c}8,4 \\
(7,2-9,8)\end{array}$ & $\begin{array}{c}5,7 \\
(4,9-6,7)\end{array}$ & $\begin{array}{c}10,0 \\
(8,8-11,3)\end{array}$ & $\begin{array}{c}5,9 \\
(5,1-6,8)\end{array}$ & $\begin{array}{c}8,0 \\
(7,1-8,9)\end{array}$ & $\begin{array}{c}6,3 \\
(5,8-6,8)\end{array}$ & $\begin{array}{c}9,1 \\
(8,6-9,6)\end{array}$ & $\begin{array}{c}1,1 \\
(1,0-1,1)\end{array}$ & $\begin{array}{c}1,0 \\
(1,0-1,1)\end{array}$ \\
\hline AVC & $\begin{array}{c}0,2 \\
(0,1-0,3)\end{array}$ & $\begin{array}{c}0,3 \\
(0,2-0,5)\end{array}$ & $\begin{array}{c}1,5 \\
(0,9-2,4)\end{array}$ & $\begin{array}{c}0,7 \\
(0,4-1,1)\end{array}$ & $\begin{array}{c}2,6 \\
(2,0-3,3)\end{array}$ & $\begin{array}{c}1,9 \\
(1,4-2,6)\end{array}$ & $\begin{array}{c}5,3 \\
(4,6-6,2)\end{array}$ & $\begin{array}{c}4,2 \\
(3,5-5,0)\end{array}$ & $\begin{array}{c}1,5 \\
(1,3-1,7)\end{array}$ & $\begin{array}{c}1,3 \\
(1,1-1,4)\end{array}$ & $\begin{array}{c}1,3 \\
(1,2-1,4)\end{array}$ & $\begin{array}{c}1,4 \\
(1,3-1,5)\end{array}$ \\
\hline Hypertension & $\begin{array}{c}7,3 \\
(6,5-8,1)\end{array}$ & $\begin{array}{c}5,4 \\
(4,8-6,1)\end{array}$ & $\begin{array}{c}28,0 \\
(25,7-30,4)\end{array}$ & $\begin{array}{c}21,0 \\
(18,9-23,2)\end{array}$ & $\begin{array}{c}44,0 \\
(41,9-46,1)\end{array}$ & $\begin{array}{c}35,1 \\
(33,1-37,1)\end{array}$ & $\begin{array}{c}56,4 \\
(54,4-58,4)\end{array}$ & $\begin{array}{c}58,2 \\
(56,4-59,9)\end{array}$ & $\begin{array}{c}23,1 \\
(22,4-23,8)\end{array}$ & $\begin{array}{c}21,1 \\
(20,4-21,8)\end{array}$ & $\begin{array}{r}2, \\
(1,7-2,5) \ddagger \\
\text { répartition }\end{array}$ & $\begin{array}{l}1 \\
\ddagger \text { (pas de } \\
\text { par sexe) }\end{array}$ \\
\hline
\end{tabular}




\begin{tabular}{|c|c|c|c|c|c|c|c|c|c|c|c|c|}
\hline $\begin{array}{l}\text { Facteur de } \\
\text { risque }\end{array}$ & \multicolumn{2}{|c|}{$18-49$ ans } & \multicolumn{2}{|c|}{$50-59$ ans } & \multicolumn{2}{|c|}{$60-69$ ans } & \multicolumn{2}{|c|}{70 ans et plus } & \multicolumn{2}{|c|}{ Population totale } & \multicolumn{2}{|c|}{$\begin{array}{l}\text { HR ajusté (IC à } 95 \% \text { ) } \\
\text { pour les formes graves } \\
\text { de la CoVID-19* }\end{array}$} \\
\hline Démence§ & $\mathrm{F}$ & $\mathrm{F}$ & $\mathrm{F}$ & $\mathrm{F}$ & $\begin{array}{c}0,8 \\
(0,4-1,6)\end{array}$ & $\begin{array}{c}0,6 \\
(0,3-1,1)\end{array}$ & $\begin{array}{c}2,9 \\
(2,3-3,7)\end{array}$ & $\begin{array}{c}3,3 \\
(2,6-4,2)\end{array}$ & $\begin{array}{c}0,9 \\
(0,7-1,2)\end{array}$ & $\begin{array}{c}1,0 \\
(0,8-1,2)\end{array}$ & $\begin{array}{c}2,7 \\
(2,5-2,9)\end{array}$ & $\begin{array}{c}2,3 \\
(2,1-2,5)\end{array}$ \\
\hline Blanc & $\begin{array}{c}65,4 \\
(63,8-67,0)\end{array}$ & $\begin{array}{c}63,7 \\
(62,2-65,2)\end{array}$ & $\begin{array}{c}77,1 \\
(74,7-79,3)\end{array}$ & $\begin{array}{c}77,0 \\
(74,6-79,1)\end{array}$ & $\begin{array}{c}83,1 \\
(81,0-85,1)\end{array}$ & $\begin{array}{c}85,0 \\
(83,3-86,6)\end{array}$ & $\begin{array}{c}85,8 \\
(84,3-87,2)\end{array}$ & $\begin{array}{c}87,8 \\
(86,3-89,1)\end{array}$ & $\begin{array}{c}72,9 \\
(71,8-73,9)\end{array}$ & $\begin{array}{c}73,2 \\
(72,2-74,1)\end{array}$ & $\begin{array}{c}1,0 \\
(1,0-1,0)\end{array}$ & $\begin{array}{c}1,0 \\
(1,0-1,0)\end{array}$ \\
\hline $\begin{array}{l}\text { Sud- } \\
\text { asiatique }\end{array}$ & $\begin{array}{c}7,2 \\
(6,3-8,1)\end{array}$ & $\begin{array}{c}6,7 \\
(5,9-7,7)\end{array}$ & $\begin{array}{c}4,7 \\
(3,4-6,4)\end{array}$ & $\begin{array}{c}3,6 \\
(2,5-5,3)\end{array}$ & $\begin{array}{c}3,5 \\
(2,4-5,0)\end{array}$ & $\begin{array}{c}1,4 \\
(0,9-2,1)\end{array}$ & $\begin{array}{c}3,9 \\
(2,9-5,2)\end{array}$ & $\begin{array}{c}1,5 \\
(1,0-2,1)\end{array}$ & $\begin{array}{c}5,7 \\
(5,1-6,4)\end{array}$ & $\begin{array}{c}4,5 \\
(4,0-5,1)\end{array}$ & $\begin{array}{c}2,0 \\
(1,8-2,3)\end{array}$ & $\begin{array}{c}1,9 \\
(1,6-2,2)\end{array}$ \\
\hline $\begin{array}{l}\text { Identité } \\
\text { autochtone }\end{array}$ & $\begin{array}{c}4,0 \\
(3,6-4,6)\end{array}$ & $\begin{array}{c}4,0 \\
(3,6-4,5)\end{array}$ & $\begin{array}{c}3,3 \\
(2,7-4,1)\end{array}$ & $\begin{array}{c}4,1 \\
(3,3-5,0)\end{array}$ & $\begin{array}{c}3,1 \\
(2,3-4,1)\end{array}$ & $\begin{array}{c}2,7 \\
(2,2-3,3)\end{array}$ & $\begin{array}{c}1,6 \\
(1,3-2,1)\end{array}$ & $\begin{array}{c}1,4 \\
(1,2-1,7)\end{array}$ & $\begin{array}{c}3,4 \\
(3,1-3,8)\end{array}$ & $\begin{array}{c}3,4 \\
(3,1-3,7)\end{array}$ & \multicolumn{2}{|c|}{$\begin{array}{c}2,4 \\
(2,2-2,7) \text { pas de } \\
\text { répartition par sexe }\end{array}$} \\
\hline Autre & $\begin{array}{c}9,4 \\
(8,4-10,4)\end{array}$ & $\begin{array}{c}9,4 \\
(8,5-10,4)\end{array}$ & $\begin{array}{c}5,0 \\
(3,9-6,3)\end{array}$ & $\begin{array}{c}5,9 \\
(4,6-7,5)\end{array}$ & $\begin{array}{c}3,2 \\
(2,5-4,2)\end{array}$ & $\begin{array}{c}3,7 \\
(2,8-4,9)\end{array}$ & $\begin{array}{c}3,6 \\
(2,9-4,5)\end{array}$ & $\begin{array}{c}2,9 \\
(2,1-3,9)\end{array}$ & $\begin{array}{c}6,9 \\
(6,3-7,5)\end{array}$ & $\begin{array}{c}6,8 \\
(6,3-7,4)\end{array}$ & $\begin{array}{c}2,1 \\
(1,8-2,4)\end{array}$ & $\begin{array}{c}1,9 \\
(1,6-2,2)\end{array}$ \\
\hline \multicolumn{13}{|c|}{ 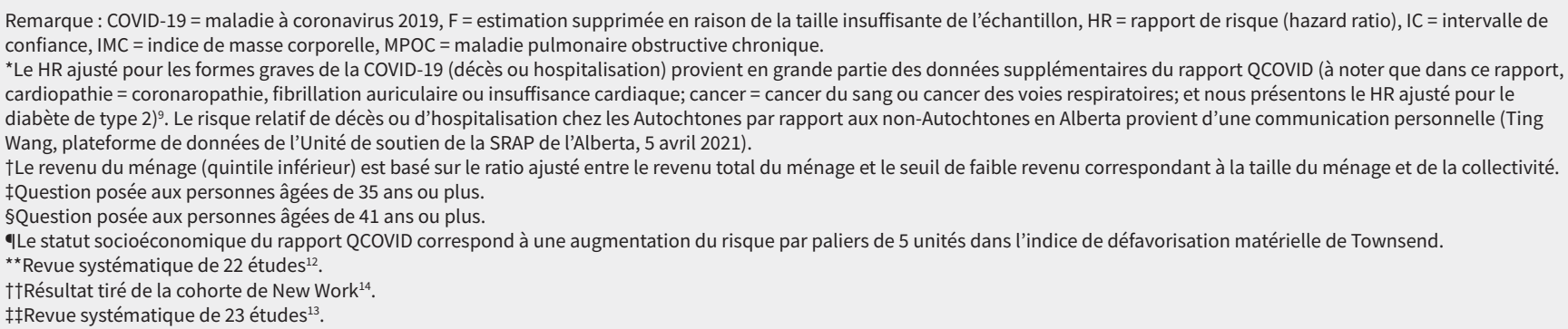 } \\
\hline
\end{tabular}

absolu de développer une forme grave de la COVID-19 pour chaque patient en fonction de ses caractéristiques individuelles, l'étude justificative ${ }^{9}$ présente également pour chaque facteur des rapports de risque (hazard ratios, ou HR) ajustés selon le sexe pour le décès ou l'hospitalisation (tableau 1).

Nous avons utilisé les données de l'ESCC et de l'ECMS, car ces enquêtes sur la santé de la population ont été menées par Statistique Canada dans l'ensemble des provinces et des territoires et sont représentatives de $97 \%$ de la population du pays $^{15,16}$. Bien que ces enquêtes excluent les personnes vivant en établissement, dans des réserves ou dans des régions éloignées et les membres à temps plein des Forces canadiennes, ces groupes ont déjà été pris en compte dans les plans de vaccination provinciaux et territoriaux actuels. Après avoir exclu 462 personnes enceintes (pour éviter d'inclure à tort des problèmes de santé liés à la grossesse comme facteurs de risque), nous avons analysé les données correspondant à 60742 participants adultes à l'ESCC de 2019 et à 6662 participants adultes à l'ECMS de 2016-2019 (pour les méthodes détaillées, consulter l'annexe 1 , accessible en anglais au www.cmaj.ca/lookup/doi/10.1503/cmaj.210529/ tab-related-content).

Une liste complète des facteurs de risque pertinents inclus dans l'ESCC est fournie dans le tableau 1. Dans toutes les provinces, les facteurs de risque les plus fréquents étaient l'hypertension ( $23,1 \%$ des hommes et $21,1 \%$ des femmes) et l'obésité ( $21,7 \%$ des hommes et 20,2\% des femmes). Environ $27 \%$ des hommes et des femmes étaient racisés, et 3,4\% s'identifiaient comme des membres des Premières Nations, des Métis ou des Inuits. Les maladies hépatiques et rénales chroniques (dont le HR ajusté de l'analyse QCOVID s'étend de 1,2 à 6,7 , selon la gravité) ne sont pas évaluées dans l'ESCC, mais moins de $3 \%$ des répondants de l'ECMS ont déclaré être atteints de l'une ou l'autre de ces maladies. Comme il n'est pas possible de lier les données de l'ESCC et de l'ECMS, nous n'avons pas pu déterminer la proportion de ces personnes qui présentaient également les comorbidités à l'étude dans l'ESCC.

Ainsi, parmi les personnes vivant dans la collectivité au Canada, $74,0 \%$ des hommes et $70,9 \%$ des femmes ont déclaré présenter au moins un facteur de risque associé à une forme 
grave de la COVID-19, et 39,3\% et 36,3\% d'entre eux, respectivement, présentaient au moins 2 facteurs de risque (voir le tableau 1 et l'annexe 2, accessible en anglais au www.cmaj.ca/ lookup/doi/10.1503/cmaj.210529/tab-related-content). Bien que la proportion de Canadiens présentant des facteurs de risque (en particulier des facteurs de risque multiples) ait augmenté avec l'âge, il est important de noter que même chez les adultes âgés de 18-49 ans, 70,9\% des hommes et $67,9 \%$ des femmes présentaient au moins 1 facteur de risque et $31 \%$ présentaient au moins 2 facteurs de risque associés à une forme grave de la COVID-19.

La fréquence de tous les facteurs de risque était plus élevée chez les hommes que chez les femmes, sauf pour ce qui est de la maladie pulmonaire obstructive chronique et de l'asthme et chez les personnes ayant un faible revenu (tableau 1). Bien que notre analyse porte sur les estimations pour l'ensemble du Canada, on peut obtenir une ventilation plus détaillée par province basée sur l'ESCC de 2017-2018 directement auprès de Statistique Canada.

\section{Quelles sont les limites de l'utilisation de l'ESCC et de l'ECMS pour évaluer les facteurs de risque associés à la COVID-19?}

Bien que l'ESCC et l'ECMS soient des enquêtes sur la santé représentatives à l'échelle nationale, leur utilisation pour estimer les facteurs de risque associés à une forme grave de la COVID-19 comporte certaines limites. Par exemple, la fréquence des facteurs de risque que nous rapportons correspond à des sous-estimations prudentes autodéclarées qui ne tiennent pas compte des maladies chroniques non diagnostiquées. Nous n'avons pas non plus été en mesure de rapporter les données relatives à certains facteurs de risque rares mais importants associés à une forme grave de la COVID-19, qui n'ont été inclus dans aucune des 2 enquêtes, tels que la trisomie 21 (HR ajusté pour les formes graves de la COVID-19 dans l'étude QCOVID 9,6 chez les hommes et 7,5 chez les femmes), l'anémie falciforme (HR ajusté 4,5 chez les hommes et 6,8 chez les femmes), les maladies neuromusculaires (HR ajusté 3,0 chez les hommes et 2,4 chez les femmes), une greffe récente de moelle osseuse ou de cellules souches (HR ajusté 1,7 chez les hommes et 1,6 chez les femmes) ou une chimiothérapie en cours (HR ajusté de 1,7-4,3 chez les hommes et de 2,1-15,0 chez les femmes, selon le traitement) ${ }^{9}$.

Bien que plusieurs études ${ }^{9,11}$ aient suggéré que les minorités racisées présentent un risque plus élevé de contracter une forme grave de la COVID-19, il est difficile de déterminer dans quelle mesure ce risque accru est dû à des facteurs biologiques ou à l'influence des déterminants sociaux de la santé. Comme l'ESCC ou l'ECMS n'incluaient pas les personnes vivant dans des réserves et que les estimations pour les territoires n'étaient pas disponibles pour une seule année de l'ESCC, il y avait dans nos données une sous-estimation des personnes s'identifiant comme des membres des Premières Nations, des Métis et des Inuits. Par ailleurs, aucune des 2 enquêtes n'a recueilli de renseignements sur les conditions de travail (par exemple, pour préciser si les personnes étaient des travailleurs essentiels ou travaillaient dans des environnements où les contacts étroits sont inévitables). Enfin, les 2 enquêtes excluaient les personnes en situation d'itinérance et celles vivant dans des établissements de soins de longue durée ou dans des prisons; ces conditions de vie constituent également un facteur de risque associés à une forme grave de la COVID-19 ${ }^{18}$, mais ces groupes ont déjà été définis comme prioritaires dans les orientations du $\mathrm{CCNI}$ en matière de vaccination.

\section{Quels facteurs devraient guider le déploiement de la vaccination contre le SRAS-CoV-2 au Canada?}

Puisque les données basées sur la population montrent que près des trois quarts des adultes canadiens présentent au moins 1 facteur de risque associé à une forme grave de la COVID-19 et que plus d'un tiers présentent au moins 2 facteurs de risque (ce qui correspond à l'analyse des données de l'enquête américaine $\mathrm{NHANES}^{6}$, on ne peut pas fonder une stratégie de vaccination uniquement sur l'âge et la présence d'un facteur de risque médical. Si un déploiement stratégique basé sur les risques est choisi en raison des limites externes quant au rythme de vaccination, les décideurs politiques devront peut-être pondérer l'importance accordée à chaque facteur de risque (déterminée à partir d'études internationales sur les risques comme l'analyse QCOVID) ${ }^{9-14}$ en fonction des données sur leur prévalence locale (qui diffère en fonction des facteurs démographiques et socioéconomiques) et du risque d'exposition (taux de transmission professionnelle ou géographique, par exemple). Notre analyse fournit des renseignements complémentaires aux données administratives sur la santé pour les décideurs politiques qui tentent de planifier l'attribution stratégique des vaccins.

Si on porte notre regard à l'étranger, on pourra s'inspirer de l'exemple d'Israël, qui a mis sur pied un programme simple et rapide (115 doses administrées pour 100 personnes en date du 30 mars 2021) et de celui du Royaume-Uni, qui a exécuté un déploiement plus complexe basé sur les risques (51 doses administrées pour 100 personnes) ${ }^{19}$. Au 30 mars 2021, $61 \%$ des gens en Israël avaient reçu une première dose de vaccin et $55 \%$ étaient complètement vaccinées; au Royaume-Uni, $45 \%$ des gens avaient reçu une première dose de vaccin et $6 \%$ étaient complètement vaccinées ${ }^{19}$. La campagne de vaccination de masse en Israël a ciblé en priorité les personnes de plus de 60 ans, puis elle a été étendue à tous les adultes. Au RoyaumeUni, le déploiement se fait plutôt par strates de risque (établies selon le score QCOVID et la tranche d'âge). Au Canada, l'approche actuelle varie considérablement : les territoires procèdent à une vaccination de masse des adultes par communauté, sans stratification des facteurs de risque, alors que certaines provinces ont mis en place des matrices plus complexes de priorisation, qui peuvent ou non inclure le milieu de travail, les problèmes de santé chroniques (les définitions et la stratégie de déploiement différant selon les provinces) et le risque de transmission dans le quartier ou la collectivité. 
Notre analyse, qui montre que la majorité des Canadiens présentent au moins un facteur de risque associé à une forme grave de la COVID-19, appuie les arguments en faveur d'une prolongation de l'intervalle entre la première et la deuxième dose de vaccin afin de réduire le nombre de doses à garder en réserve et de maximiser ainsi le nombre de premières doses administrées, dans la mesure où on continue d'observer que la protection conférée par la première dose est élevée ${ }^{4}$. Une analyse de décision récemment publiée aux États-Unis semble indiquer qu'on pourrait éviter de $23 \%$ à $32 \%$ des cas de COVID-19 en appliquant une stratégie de déploiement d'une seule dose de vaccin, au lieu de conserver $50 \%$ des stocks de vaccins pour une deuxième dose 3 ou 4 semaines plus $\operatorname{tard}^{20}$. Une autre étude de modélisation a confirmé que cette hypothèse était valable, même si l'efficacité d'une seule dose était aussi faible que $55 \%{ }^{21}$. Fait encourageant : les données préliminaires suggèrent que l'efficacité d'une seule dose de vaccin en conditions réelles pourrait être de l'ordre de $80 \%{ }^{22-25}$. De nouvelles données ${ }^{26}$ suggèrent également que, pour la plupart des gens, l'immunogénicité après une première dose de vaccin serait suffisante pour justifier d'allonger l'intervalle entre les 2 doses. L'émergence de variants sous surveillance à l'origine de la troisième vague de la pandémie est un autre argument en faveur de l'offre rapide d'une première dose à l'ensemble de la population, avec une surveillance continue de son efficacité ${ }^{4}$. Ainsi, le 30 décembre 2020, le Royaume-Uni a instauré une approche consistant à retarder l'administration de la deuxième dose, et le CCNI a fait de même le $1^{\text {er }}$ mars 2021; les CDC ont quant à eux autorisé un délai allant jusqu'à 6 semaines pour les deuxièmes doses de vaccins à ARNm le 21 janvier 2021. Notons toutefois qu'une mise en garde s'impose à l'égard de cette approche : il n'existe pas de données sur la durabilité de la protection conférée par la première dose pour certains sousgroupes, comme les personnes âgées, les personnes immunodéprimées ou les patients atteints d'un cancer ${ }^{27}$; or, il se pourrait que la réplication virale prolongée chez les personnes immunodéprimées favorise l'émergence d'un plus grand nombre de variants du SRAS-CoV-228.

Notre analyse permet non seulement d'informer les Canadiens qui se demandent combien de personnes se trouvent dans la même strate de risque qu'eux, mais aussi de souligner l'importance de continuer à suivre les recommandations de la santé publique, telles que le port du masque universel, la distanciation physique et le lavage fréquent des mains durant le déploiement des programmes de vaccination, dans la mesure où la plupart des adultes au Canada présentent au moins un facteur de risque associé à une forme grave de la COVID-19.

\section{Conclusion}

Puisque l'optimisation du déploiement des vaccins contre le SRAS-CoV-2 est cruciale pour éviter la morbidité et la mortalité évitables associées à la COVID-19, les plans actuels sont scrutés à la loupe. Au Canada comme ailleurs dans le monde, on cherche à établir un équilibre entre la rapidité du déploiement et la complexité et les défis que pourrait poser l'application d'une stratégie fondée sur les risques. Notre analyse des données d'enquêtes nationales basées sur la population semble indiquer que les facteurs de risque associés à une forme grave de la COVID-19 sont tellement répandus dans la population qu'il serait peu efficace de les utiliser pour orienter une stratégie de vaccination. Une évaluation plus détaillée des risques médicaux, géographiques et professionnels pourrait être nécessaire en cas de contraintes d'approvisionnement. Puisque la troisième vague de la pandémie de COVID-19 semble toucher de manière disproportionnée les travailleurs essentiels des quartiers économiquement défavorisés, la prise en compte de ces facteurs de risque pourrait être nécessaire pour atteindre les objectifs d'équité visés ${ }^{29}$. L'établissement des priorités pour la vaccination est une question d'éthique, mais aussi de science. À ce titre, nous considérons que la transparence du processus décisionnel est importante, en particulier lorsque les décisions varient d'une région à l'autre, afin de démontrer que les raisonnements qui sous-tendent les décisions sont fondés sur des données et concordent avec les valeurs communes aux populations locales.

\section{Références}

1. Ismail SJ, Zhao L, Tunis MC, et al.; National Advisory Committee on Immunization. Key populations for early COVID-19 immunization: preliminary guidance for policy. CMAJ 2020;192:E1620-32.

2. National Advisory Committee on Immunization. Recommendations on the use of COVID-19 vaccines. Ottawa: Government of Canada; 2021. Accessible ici : www.canada.ca/en/public-health/services/immunization/national-advisory -committee-on-immunization-naci/recommendations-use-covid-19-vaccines. html (consulté le 30 mars 2021).

3. Bubar KM, Reinholt K, Kissler SM, et al. Model-informed COVID-19 vaccine prioritization strategies by age and serostatus. Science 2021;371:916-21.

4. Kadire SR, Wachter RM, Lurie N. Delayed second dose versus standard regimen for COVID-19 vaccination. N Engl J Med 2021;384:e28.

5. Vaccines for COVID-19: How to get vaccinated or register. Ottawa: Government of Canada; 2021. Accessible ici : www.canada.ca/en/public-health/services/ diseases/coronavirus-disease-covid-19/vaccines/how-vaccinated.html\#a5 (consulté le 7 avril 2021).

6. Li HL, Cheung BMY. The proportion of adult Americans at risk of severe COVID19 illness. J Gen Intern Med 2021;36:259-61.

7. Manuel DG, Rosella LC, Stukel TA. Importance of accurately identifying disease in studies using electronic health records. BMJ 2010;341:c4226.

8. McAlister FA, Tonelli M, Wiebe N, et al. The ecology of medical care for adults in Alberta, 2002/03 to 2016/17: a retrospective cohort study. CMAJ Open 2020;8:E169-77.

9. Clift AK, Coupland $\mathrm{CAC}, \mathrm{Keogh} \mathrm{RH}$, et al. Living risk prediction algorithm (QCOVID) for risk of hospital admission and mortality from coronavirus 19 in adults: national derivation and validation study. BMJ 2020;371:m3731.

10. Lowe KE, Zein J, Hatipoglu U, et al. Association of smoking and cumulative pack-year exposure with COVID-19 outcomes in the Cleveland Clinic COVID-19 Registry. JAMA Intern Med 2021 Jan. 25 [cyberpublication avant impression]. doi: 10.1001/jamainternmed.2020.8360.

11. Wingert A, Pillay J, Gates M, et al. Risk factors for severe outcomes of COVID-19: a rapid review. medRxiv 2020 Sept. 1. doi: 10.1101/2020.08.27.201834344.

12. Chu Y, Yang J, Shi J, et al. Obesity is associated with increased severity of disease in COVID-19 pneumonia: a systematic review and meta-analysis. Eur $J$ Med Res 2020;25:64.

13. Fang X, Li S, Yu H, et al. Epidemiological, comorbidity factors with severity and prognosis of COVID-19: a systematic review and meta-analysis. Aging (Albany NY) 2020;12:12493-503.

14. Petrilli CM, Jones SA, Yang J, et al. Factors associated with hospital admission and critical illness among 5279 people with coronavirus disease 2019 in New York City: prospective cohort study. BMJ 2020;369:m1966.

15. Canadian Community Health Survey - Annual Component (CCHS). Ottawa: Statistics Canada; modified 2019 Nov. 18. Accessible ici : www23.statcan.gc.ca/ imdb/p2SV.pl?Function=getSurvey\&ld=1208978 (consulté le 14 mars 2021). 
16. Canadian Health Measures Survey (CHMS). Ottawa: Statistics Canada; modified 2019 Dec. 4. Accessible ici : www23.statcan.gc.ca/imdb/p2SV. pl?Function=getSurvey\&SDDS=5071 (consulté le 14 mars 2021).

17. Table 13-10-0777-01 Number and percentage of adults (aged 18 years and older) in the household population with underlying health conditions, by age and sex (two-year period). Ottawa: Statistics Canada; 2021. Accessible ici : www150. statcan.gc.ca/t1/tbl1/en/tv.action?pid=1310077701 (consulté le 7 avril 2021).

18. Richard L, Booth R, Rayner J, et al. Testing, infection and complication rates of COVID-19 among people with a recent history of homelessness in Ontario, Canada: a retrospective cohort study. CMAJ Open 2021;9:E1-9.

19. Roser M, Ritchie H, Ortiz-Ospina E, et al. Coronavirus (COVID-19) vaccinations. Oxford: Our World in Data; 2021. Accessible ici : https://ourworldindata.org/ covid-vaccinations (consulté le 30 mars 2021).

20. Tuite AR, Zhu L, Fisman DN, et al. Alternative dose allocation strategies to increase benefits from constrained COVID-19 vaccine supply. Ann Intern Med 2021 Jan. 5 [cyberpublication avant impression]. doi: 10.7326/M20-8137.

21. Paltiel AD, Zheng A, Schwartz JL. Speed versus efficacy: quantifying potential tradeoffs in COVID-19 vaccine deployment. Ann Intern Med 2021 Jan. 5 [cyberpublication avant impression]. doi: 10.7326/M20-7866.

22. Torjesen I. COVID-19: first doses of vaccines in Scotland led to a substantial fall in hospital admissions. BMJ 2021;372:n523.

23. Iacobucci G. COVID-19: Single dose of Pfizer and Oxford vaccines cuts risk of hospital admission by $80 \%$ in over 80 s, data suggest. BMJ 2021;372:n612.

24. Rinott E, Youngster I, Lewis YE. Reduction in COVID-19 patients requiring mechanical ventilation following implementation of a national COVID-19 vaccination program: Israel, December 2020-February 2021. MMWR Morb Mortal Wkly Rep 2021;70:326-8.

25. Thompson MG, Burgess JL, Naleway AL, et al. Interim estimates of vaccine effectiveness of BNT162b2 and mRNA-1273 COVID-19 vaccines in preventing SARS-CoV-2 infection among health care personnel, first responders, and other essential and frontline workers: eight U.S. locations, December 2020March 2021. MMWR Morb Mortal Wkly Rep 2021;70:495-500.

26. Voysey M, Costa Clemens SA, Madhi SA, et al.; Oxford COVID Vaccine Trial Group. Single-dose administration and the influence of the timing of the booster dose on immunogenicity and efficacy of ChAdOx1 nCoV-19 (AZD1222) vaccine: a pooled analysis of four randomised trials [erratum publié dans Lancet 2021;397:880]. Lancet 2021;397:881-91.

27. Monin-Aldama L, Laing AG, Muñoz-Ruiz M, et al. Interim results of the safety and immune-efficacy of 1 versus 2 doses of COVID-19 vaccine BNT162b2 for cancer patients in the context of the UK vaccine priority guidelines. medRxiv 2021 Mar. 17. doi: 10.1101/2021.03.17.21253131.

28. Kemp SA, Collier DA, Datir RP, et al. SARS-CoV-2 evolution during treatment of chronic infection. Nature 2021 Feb. 5 [cyberpublication avant impression]. doi: 10.1038/s41586-021-03291-y.

29. Chagla Z, Ma H, Sander B, et al. Characterizing the disproportionate burden of SARS-CoV-2 variants of concern among essential workers in the Greater Toronto Area, Canada. medRxiv 2021 Mar. 26. doi: 10.1101/2021.03.22.21254127.
Intérêts concurrents : Lynora Saxinger est membre du comité de planification en contexte de pandémie de l'EZMSA de l'Association médicale de l'Alberta (à titre bénévole) et déclare avoir reçu des honoraires pour une présentation sur la maladie à coronavirus 2019 lors d'un congrès sur les soins virtuels contre le cancer. Aucun autre intérêt concurrent n'a été déclaré.

Cet article a été révisé par des pairs.

Affiliations : Département de médecine (McAlister, Saxinger), Faculté de médecine et de dentisterie, Université de l'Alberta, Edmonton, Alb.; Division de l'analyse de la santé (Bushnik), Statistique Canada, Ottawa, Ont.; Département de médecine (Leung), École de médecine Cumming, Université de Calgary, Calgary, Alb.; Alberta Health Services (Saxinger), Edmonton, Alb.

Collaborateurs : Tous les auteurs ont contribué à l'élaboration et à la conception de l'étude. Tracey Bushnik a recueilli et analysé les données, sous la supervision de Finlay McAlister, d'Alexander Leung et de Lynora Saxinger. Finlay McAlister a rédigé la première ébauche du manuscrit et tous les auteurs en ont révisé de façon critique le contenu intellectuel. Tous les auteurs ont donné leur approbation finale pour la version soumise pour publication et assument l'entière responsabilité de tous les aspects du travail.

Financement : Finlay McAlister est détenteur de la Chaire de recherche en médecine cardiovasculaire des Services de santé de l'Alberta.

Propriété intellectuelle du contenu : Il s'agit d'un article en libre accès distribué conformément aux modalités de la licence Creative Commons Attributions (CC BY-NC-ND 4.0), qui permet l'utilisation, la diffusion et la reproduction dans tout médium à la condition que la publication originale soit adéquatement citée, que l'utilisation se fasse à des fins non commerciales (c.-à-d., recherche ou éducation) et qu'aucune modification ni adaptation n'y soit apportée. Voir : https://creativecommons.org/licenses/by-nc-nd/4.0/deed.fr.

Remerciements : Les auteurs remercient $M^{\text {me }}$ Ting Wang, de I'Unité de soutien de la SRAP de l'Alberta, d'avoir fourni des données non publiées sur les taux relatifs de décès ou d'hospitalisation chez les Autochtones par rapport aux nonAutochtones de l'Alberta (5 avril 2021).

Correspondance : Finlay McAlister, finlay.mcalister@ualberta.ca 